\title{
Detection of the Spontaneous Change in the Burgers Vector of Perfect Dislocation Loops in Iron
}

\author{
K. Arakawa, M. Hatanaka, H. Mori
}

Research Center for Ultra-High Voltage Electron Microscopy, Osaka University, Yamada-Oka, Suita, Osaka 565-0871, Japan

Two types of interstitial-type perfect dislocation loops are formed in ferritic alloys upon irradiation with energetic particles - those with the Burgers vector of $1 / 2<111>$ and those with the Burgers vector of $<100>$ (see Ref. [1]). The mobility of the one-dimensional prismatic glide of $1 / 2<111>$ loops is extremely higher than that of $<100>$ loops [2]. Therefore, such a difference between the values of the Burgers vector will be significant to the microstructural evolution upon irradiation. In this study, we investigate the dynamic behavior of loops with diameters ranging from a few to a few hundred nanometers in pure $\alpha-\mathrm{Fe}$ by using in situ TEM. In particular, we present the first observation on spontaneous change in the Burgers vector of small loops-a process that cannot be explained by the conventional dislocation theory [3].

Pure bcc Fe (99.999\%) supplied by Showa Denko Inc. was used as the specimen. It was rolled into 0.08-mm-thick sheets. These sheets were pre-annealed at $1120 \mathrm{~K}$ for $1 \mathrm{~h}$ under a hydrogen atmosphere and electrochemically polished for TEM. We were able to observe the prismatic glide motion of loops and the process of change in their Burgers vector by the following two techniques: (1) high-energy electron irradiation upon which loops were formed due to knock-on displacements of Fe atoms by incident electrons and (2) thermal annealing. High-energy electron irradiation was performed in a high-voltage electron microscope H-3000 (Hitachi) operated at an acceleration voltage of $1000 \mathrm{kV}$, and the behavior of the loops was simultaneously observed. The beam fluxes were primarily $1 \times 10^{24} \mathrm{e}^{-/ \mathrm{m}^{2}} \mathrm{~s}$ and $1 \times 10^{23} \mathrm{e}^{-/} \mathrm{m}^{2} \mathrm{~s}$, and the irradiation temperatures ranged from 110 to $290 \mathrm{~K}$. Thermal annealing and in situ observations were performed using a general-use microscope JEM-2010 (JEOL) at an acceleration voltage of $200 \mathrm{kV}$ to prevent the introduction of additional knock-on displacements into the specimens. The annealing was performed following the induction of the loops by high-energy electron irradiation with irradiation doses ranging from $1 \times$ $10^{26}$ to $1 \times 10^{27} \mathrm{e}^{-} / \mathrm{m}^{2}$ at temperatures ranging from 110 to $190 \mathrm{~K}$. The annealing temperature ranged from 290 to $900 \mathrm{~K}$. Bright-field imaging was used in the in situ TEM observations. The images were recorded using a CCD camera with a time resolution of $1 / 30 \mathrm{~s}$.

The projected direction of motion of a loop spontaneously changed from [110] to [1 $\overline{1} 0]$ along the observation axis of approximately [001]; this implies that the Burgers vector spontaneously changes from $1 / 2[111]$ to $1 / 2[1 \quad 1]$. In the image shown in Fig. 1, the marked loop changes spontaneously. The loop was identified to be $1 / 2[\overline{1} 11](1 \overline{1} 0)$ prior to the change and $[010](010)$ after the change by the image calculation of circular loops for various combinations of the Burgers vectors and habit planes using the Howie and Whelan method [4] upon a two-beam condition. These phenomena were observed for small $1 / 2<111>$ loops, the diameters of which were less than approximately 20 to 30 $\mathrm{nm}$. In contrast, the Burgers vector of small mobile $<100>$ loops and larger $1 / 2<111>$ loops rarely changed spontaneously. 
Recently, extensive molecular dynamics calculations have suggested that small loops comprising several to several tens self-interstitial atoms (SIAs) have the structure of bundles of crowdions (see Ref. [5]). The origin of spontaneous change in the Burgers vector of the $1 / 2<111>$ loops can be understood by the idea that these loops comprise bundles of crowdions. Upon energetic-particle irradiation and thermal annealing of a $1 / 2<111>$ loop comprising a bundle of crowdions along the closest-packed direction of the $<111>$ axes, any of the atoms in a crowdion of the loop can be easily displaced from the original crowdion axis by frequent atomic vibration, thereby rotating the crowdion axis to another $<111>$ or the second-closest packed $<100>$ axes. If this rotation of the crowdion axes propagates through the loop, the Burgers vector spontaneously changes from $1 / 2<111>$ or $<100>$. In the $<100>$ loops, the interatomic distance along the $<100>$ crowdion axes is greater; therefore, a rotation of crowdion axes will rarely occur. This probably leads to the difficulty encountered with regard to the spontaneous change in their Burgers vector, which is the origin of the stability of energetically unfavorable $<100>$ loops [6]. Thus, the spontaneous change in the Burgers vector strongly suggests that a loop comprising as many as several thousand to a few ten thousand SIAs is a bundle of crowdions.

\section{References}

[1] B. L. Eyre and R. Bullough, Philos. Mag. 12 (1965) 31.

[2] K. Arakawa, M. Hatanaka, H. Mori, and K. Ono, J. Nucl. Mater. 329-333 (2004) 1194.

[3] K. Arakawa, et al., submitted to Phys. Rev. Lett.

[4] A. Howie and M. J. Whelan, Proc. R. Soc. London Ser. A 263 (1961) 217.

[5] Yu. N. Osetsky, et al., Phil. Mag. 83 (2003) 61.

[6] J. Marian and B. D. Wirth, Phys. Rev. Lett. 88 (2002) 255507.

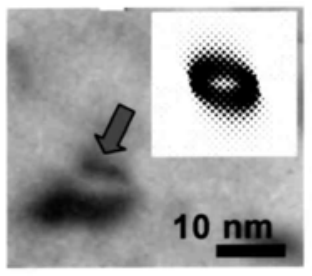

$0.00 \mathrm{~s}$

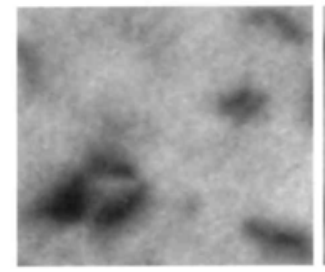

$0.17 \mathrm{~s}$

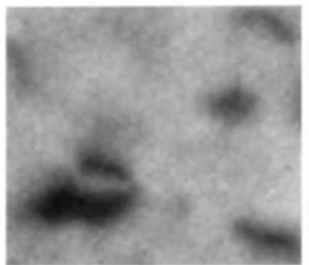

$0.07 \mathrm{~s}$

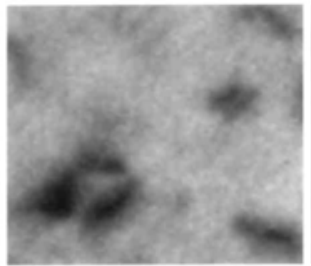

$0.20 \mathrm{~s}$

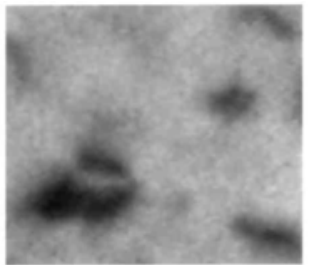

$0.10 \mathrm{~s}$

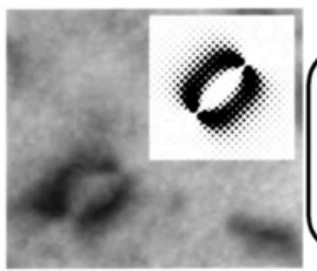

$0.30 \mathrm{~s}$

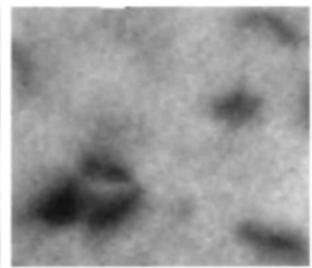

$0.13 \mathrm{~s}$

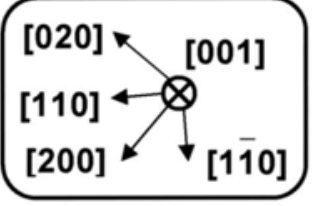

110]

FIG. 1. Spontaneous change in the TEM image of a dislocation loop upon thermal annealing at 570 $\mathrm{K}$. The reflection $\mathbf{g}=020$ was adopted. The inserted images are the calculated ones by using the Howie-Whelan method. 